Reprod. Nutr. Dévelop., 1986, 26 (1 B), 339-340.

\title{
Évolution de la morphologie de la muqueuse intestinale et du renouvelle- ment cellulaire de son épithélium chez l'agneau préruminant
}

D. ATTAIX, J.-C. MESLIN (*)

Laboratoire d'Etude du Métabolisme Azoté,

I.N.R.A., Theix, 63122 Ceyrat, France.

(*) Station de Recherches de Nutrition,

I.N.R.A., 78350 Jouy-en-Josas, France.

Summary. The depth of crypts, the height of villi and the position of labelled cells were measured along the small intestine of one-week and five-week old preruminant lambs, at different times after an intravenous injection of tritiated thymidine. Epithelium renewal times increased with age, although there was a significant reduction in villus size at all intestinal levels.

Les mécanismes régissant le développement postnatal de la muqueuse intestinale sont mal connus. Le but de ce travail est d'établir, chez l'agneau préruminant, les relations existant entre la morphologie de la muqueuse intestinale, le renouvellement cellulaire de son épithélium et le renouvellement des protéines qui a été mesuré auparavant (Combe et al., 1979). A notre connaissance, seuls Moon et Joel (1975) ont brièvement décrit la migration des entérocytes chez le jeune agneau.

Matériel et méthodes. Dix-huit agneaux mâles préruminants croisés lle de France $\times$ Romanov-Limousin $(10$ animaux âgés d'une semaine et 8 âgés de 5 semaines), nourris exclusivement avec un lait artificiel distribué ad libitum, ont reçu une injection intraveineuse de $0,6-0,7 \mathrm{mCi}$ de thymidine tritiée $/ \mathrm{kg}$ de poids vif. Ils ont été sacrifiés $24,36,48,60$ ou $96 \mathrm{~h}$ après l'injection du marqueur à raison d'au moins 2 agneaux par temps et âge considérés. Après traitement histologique d'échantillons de duodénum proximal et distal, de jéjunum et d'iléon prélevés à l'abattage et réalisation d'autoradiographies, nous avons mesuré la profondeur des cryptes intestinales, la hauteur des villosités et la position du front de marquage des entérocytes. La conduite des animaux et les techniques mises en œuvre ont été décrites en détail auparavant (Attaix et al., 1984).

Résultats et discussion. A un âge donné, les dimensions des systèmes crypte + villosité (fig. 1) des différents agneaux ou d'un même animal sont sujettes à de grandes variations à tous les sites intestinaux. Ceci est vraisemblablement dû au remodelage postnatal de la paroi intestinale consécutif à son adaptation aux processus de la digestion. Quel que soit l'âge des agneaux, les cryptes les plus profondes sont observées dans le duodénum et les villosités les plus longues dans le jéjunum. La taille des villosités diminue avec l'âge, ce qui est en accord avec les observations de Moon et Joel (1975). Ce raccourcissement est d'autant plus marqué que le site intestinal considéré est distal et se trouve moins soumis à l'action des sécrétions digestives, principalement pancréatique et biliaire, qui exercent une action positive sur la hauteur des villosités.

La vitesse de migration des entérocytes (tabl. 1) décroît avec l'âge surtout dans le duodénum $(-67 \%)$ et de façon moins importante dans le jéjunum 
(- $48 \%$ ) et l'iléon (-36\%). Or la flore intestinale, principalement présente dans I'intestin grêle distal, exerce un effet positif sur la migration des cellules épithéliales. Moon et Joel (1975) ont également décrit une réduction de la vitesse de migration des entérocytes de l'agneau entre les âges d'un et de 21 jours.

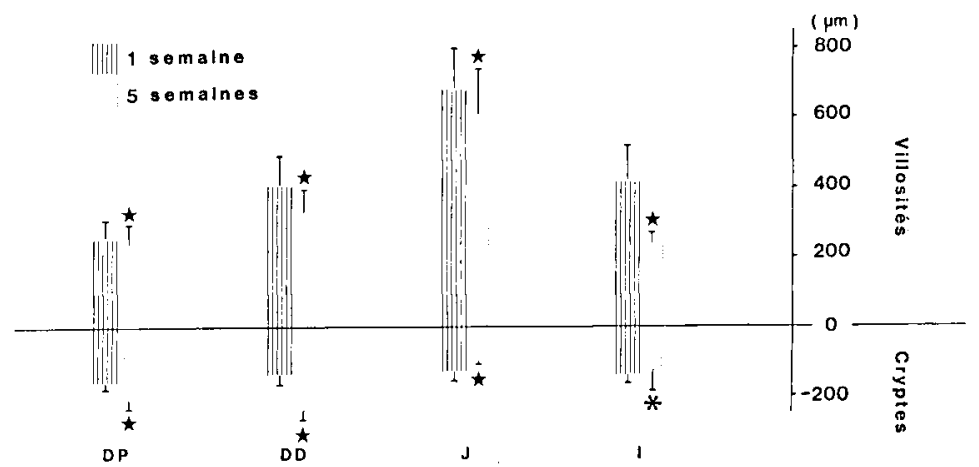

FIG. 1. - Hauteur des villosités et profondeur des cryptes intestinales chez l'agneau préruminant. Moyennes et écart-types $; \mathrm{DP}=$ Duodénum Proximal $; \mathrm{DD}=$ Duodénum Distal $; \mathrm{J}=$ Jéjunum $; 1=$ lléon: Signification des différences observées entre les 2 âges étudiés : $\star P<0,01 ; * P<0,05$.

Les temps de renouvellement de l'épithélium intestinal (tabl. 1), calculés à partir des équations des droites de régression de la position des fronts de marquage en fonction du temps, sont les plus courts dans le duodénum. Ils augmentent avec l'âge, en particulier dans le duodénum et le jéjunum. Ceci résulte de la réduction de la vitesse de migration des cellules épithéliales, bien que la taille des systèmes crypte + villosité diminue, et reste valable même lorsque la taille du compartiment prolifératif (cryptes) augmente (duodénum proximal). Ce ralentissement du renouvellement cellulaire est parallèle à celui du renouvellement des protéines observé avant le sevrage (Combe et al., 1979).

TABL. 1. - Vitesse de migration (VM) des entérocytes et estimation des temps de renouvellement (TR) de l'épithélium intestinal chez l'agneau préruminant.

\begin{tabular}{|c|c|c|c|c|}
\hline & \multicolumn{2}{|c|}{$\mathrm{VM}(\mu \mathrm{m} / \text { heure })^{*}$} & \multicolumn{2}{|c|}{ TR (heures)** } \\
\hline & $1 \mathrm{sem}$ & 5 sem & $1 \mathrm{sem}$ & $5 \mathrm{sem}$ \\
\hline Duodénum proximal & $8,5 \pm 0,3$ & $2,8 \pm 0,1$ & $49,9(45,4-54,4)$ & $108,8(95,4-122,9)$ \\
\hline Jéjunum & $8,9 \pm 0,5$ & $4,6 \pm 0,1$ & $91,5(81,8-101,1)$ & $141,9(132,9-151,2)$ \\
\hline lléon & $4,5 \pm 0,3$ & $2,9 \pm 0,1$ & $94,4(82,5-106,4)$ & $114,4(102,5-126,7)$ \\
\hline
\end{tabular}

* : \pm écart-type. ${ }^{*}$ : estimation et $($ ) limites de confiance à $95 \%$.

En conclusion, chez l'agneau en allaitement artificiel, le renouvellement cellulaire de l'épithélium intestinal diminue avec l'âge en raison d'une notable réduction de la vitesse de migration des entérocytes, bien que la taille des villosités régresse significativement à tous les sites intestinaux.

Attaix D., Meslin J. C., Combe E., 1984. Intestinal epithelium renewal time in the young lamb. Nutr. Rep. Int., 29, 689-697.

Combe E., Attaix D., Arnal M., 1979. Protein turnover in the digestive tract of the lamb throughout the development. Ann. Rech. Vet., 10, 436-439.

Moon H. W., Joel D. D., 1975. Epithelial cell migration in the small intestine of sheep and calves. Am. J. vet. Res., 36, 187-189. 